\title{
JUEZ INTELIGENTE. SISTEMA EXPERTO QUE ASISTE AL JUEZ EN LA VALORACIÓN DE LA PRUEBA JUDICIAL
}

\section{Juez inteligente. Expert system that assists the judge in the assessment of judicial proof}

\author{
Orión Vargas Vélez* \\ Universidad de Medellín
}

Abogado por la Universidad de Medellín. PhD en la Universidad Pontificia Bolivariana (2012). Profesor Titular e Investigador del Grupo de Investigaciones Jurídicas de la facultad de derecho de la Universidad de Medellín. Autor, coautor y editor de las traducciones al castellano de las obras Lo probable y lo demostrable (2017) y Un ensayo sobre la creencia y la aceptación (2021) (L. J. Cohen); Los fundamentos probatorios del razonamiento probabilístico (2016) (David A. Schum). Autor del libro El razonamiento inductivo en la valoración de la prueba judicial (2019), publicado por la universidad de Salamanca. Cocreador del sistema experto Juez Inteligente. ORCID iD: 0000-0002-9230-2908. Contacto: ovargas@udemedellin.edu.co 


\section{Resumen:}

El Juez Inteligente es un sistema experto que -con base en las credenciales de la prueba judicial (credibilidad, pertinencia y peso probatorio), las reglas de la experiencia y las reglas de la ciencia-asiste al estudiante, profesor, abogado en ejercicio, fiscal o juez, en la construcción de estructuras, argumentos o inferencias probatorias, que permiten determinar la pretensión o hipótesis que es más o menos probable en un proceso judicial. En el presente artículo, se presentan algunas aproximaciones acerca del sistema experto Juez Inteligente, con la intención de que pueda implementarse a futuro en diferentes latitudes.

\section{Abstract:}

Juez Inteligente is an expert system that -based on the credentials of evidence (credibility, relevance and inferential force or weight), rules of experience and rules of science- assists the student, professor, attorney at law, prosecutor or judge, in the construction of evidential structures, arguments or inferences, which make it possible to determine the claim or hypothesis that is more or less probable in a judicial trial. In this paper, some approximations about the Intelligent Judge expert system are presented, with the intention that it can be implemented in the future in different latitudes.

\section{Palabras clave:}

Razonamiento probatorio - Inteligencia artificial (IA) - Prueba - Valoración de la prueba

\section{Keywords:}

Evidence Based Reasoning (EBR) - Artificial Intelligence (AI) - Evidence - Assessment of Proof

\section{Sumario:}

1. Inteligencia artificial (IA) - 2. Agentes inteligentes o sistemas expertos 3. Sistema experto Juez Inteligente -4 . Entradas que deben ser asignadas por el usuario en el sistema experto Juez Inteligente - 5. Credenciales probatorias y reglas de la experiencia y/o de la ciencia que deben ser asignadas por el usuario en el sistema experto Juez Inteligente - 6. Algunas propiedades y reglas de la probabilidad baconiana -7 . Lista de referencias 


\section{INTELIGENCIA ARTIFICIAL (IA)}

Es el campo de la ciencia y la ingeniería relacionado con la teoría y la práctica del desarrollo de sistemas, que exhiben características asociadas con la inteligencia del comportamiento humano, tales como la percepción, el procesamiento del lenguaje natural, la planeación y solución de problemas, el aprendizaje y la adaptación y la actuación en el entorno (Tecuci et al., 2016a).

La comprensión de los principios que permiten el comportamiento inteligente en los seres humanos, el desarrollo de los sistemas expertos, la formalización del conocimiento, la mecanización del razonamiento en diversas áreas del quehacer humano, la facilitación del trabajo con las computadoras y el desarrollo de sistemas que asistan y/o complementen el razonamiento humano son las metas de la ciencia y la ingeniería del conocimiento actualmente. Las ciencias de la computación, las matemáticas, el lenguaje, la neurociencia, la ingeniería -en sus diversas áreas - la estadística, la lógica difusa, la economía, la cibernética, la filosofía y muchas otras disciplinas hacen parte de un mismo campo interdisciplinario en el que dichas disciplinas se intersecan (Tecuci et al., 2016b).

\section{AGENTES INTELIGENTES O SISTEMAS EXPERTOS}

Un agente inteligente o sistema experto es un sistema que percibe su entorno (por ejemplo, el mundo físico, datos que son ingresados por un usuario a través de una interfaz gráfica de usuario - GUI, en adelante-, Internet u otro entorno complejo), razona para interpretar dichas percepciones, realiza inferencias, resuelve problemas y determina las acciones a realizar sobre ese entorno para ejecutar las tareas para las que ha sido diseñado (Tecuci, Marcu et al., 2016a).

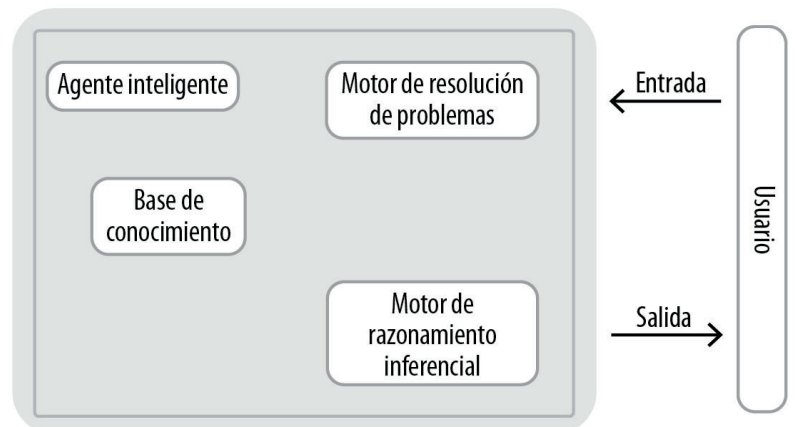

Figura 1. Arquitectura de un agente inteligente o sistema experto.

Figura 1:

Arquitectura de un agente inteligente o sistema experto. Fuente: Elaboración propia. 
La figura 1 ilustra una arquitectura básica de un agente inteligente o sistema experto:

\section{i. Base de conocimiento}

Es un tipo de memoria ${ }^{1}$ a largo plazo que contiene una estructura de datos que representan los objetos de un campo de aplicación específico, las leyes que gobiernan dichas estructuras de datos y las operaciones que pueden ser realizadas con dichas estructuras de datos.

ii. Motor de resolución de problemas

Implementa métodos generales que, empleando el conocimiento extraído de la base de conocimiento, permite la interpretación de los datos de entrada y provee una salida o respuesta apropiada en la resolución de un problema.

ii. Motor de razonamiento inferencial

Es un tipo de memoria a corto plazo donde es realizado el razonamiento probatorio o la construcción de inferencias con base en los datos de entrada, la base de conocimiento y el motor de resolución de problemas (Tecuci et al., 2016).

\section{SISTEMA EXPERTO JUEZ INTELIGENTE²}

El Juez Inteligente es un programa de computador inteligente o sistema experto que emplea una base de conocimiento y un procedimiento de construcción de inferencias para calcular el peso o valor probatorio de un conjunto ${ }^{3}$

1 "Inteligencia artificial. En informática, la memoria es un dispositivo físico que forma parte de toda computadora, capaz de grabar en ubicaciones específicas una cantidad fija de información, y recuperarla tras un tiempo determinado" (Houdé, 2003).

2 Otras investigaciones realizadas se pueden encontrar en: https://investigaciones-pure.udem. edu.co/en/persons/orión-vargas-vélez

3 Artículo 197 del Código Procesal Civil del Perú. Valoración de la prueba.

Todos los medios probatorios son valorados por el Juez en forma conjunta, utilizando su apreciación razonada. Sin embargo, en la resolución sólo serán expresadas las valoraciones esenciales y determinantes que sustentan su decisión.

Artículo 393 del Código Procesal Penal del Perú. Normas para la deliberación y votación.

(...)

2. El Juez Penal para la apreciación de las pruebas procederá primero a examinarlas individualmente y luego conjuntamente con las demás. La valoración probatoria respetará las reglas de la sana crítica, especialmente conforme a los principios de la lógica, las máximas de la experiencia y los conocimientos científicos [Énfasis agregado]. 
de pruebas en un proceso judicial. El Juez Inteligente interactúa con un usuario (estudiante, profesor, abogado en ejercicio, juez o fiscal) a través de una GUI y permite la construcción de inferencias, desde las pruebas hasta las pretensiones que son planteadas y alcanzadas por el usuario. La figura 2 ilustra los enlaces de estas inferencias entre las pretensiones, los hechos y las pruebas mediante el uso de una cadena de razonamiento simple (Wigmore, 1937) (Schum, 2016) (Anderson, et al., 2005) (Tecuci et al., 2016b) (Vargas, 2019).

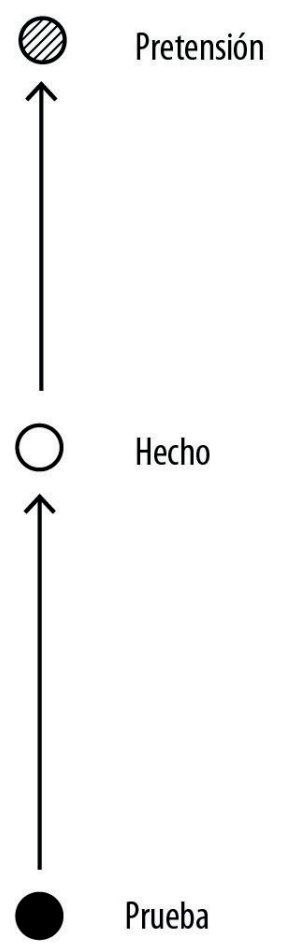

Figura 2. Cadena de razonamiento simple que ilustra los enlaces entre una pretensión, un hecho que es favorable $(f)$ a la pretensión y una prueba que es favorable $(f)$ al hecho.

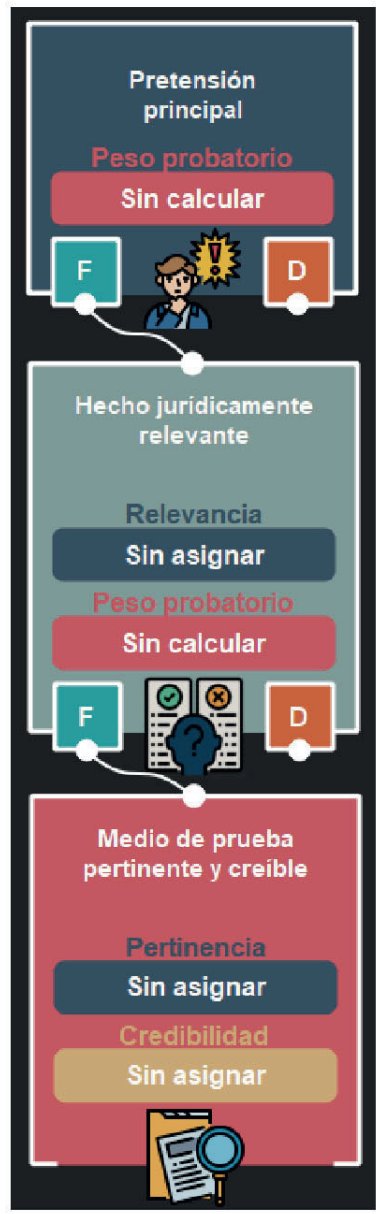

Figura 2:

Cadena de razonamiento simple que ilustra los enlaces entre una pretensión, un hecho que es favorable $(f)$ a la pretensión y una prueba que es favorable $(f)$ al hecho. Fuente: Elaboración propia. 


\section{ENTRADAS QUE DEBEN SER ASIGNADAS POR EL USUARIO EN EL SISTEMA EXPERTO JUEZ INTELIGENTE}

En un proceso civil, la parte demandante, quien tiene la carga de la prueba ${ }^{4}$ plantea: i) una pretensión principal; ii) varios hechos jurídicamente relevantes conectados favorablemente $(f)$ en relación con dicha pretensión; iii) un conjunto de pruebas pertinentes y creíbles conectadas favorablemente $(f)$ en relación con dichos hechos.

Estos tres insumos son extraídos de la demanda y la respectiva contestación de la demanda ${ }^{5}$ e ingresados al sistema experto Juez Inteligente una vez que el juez ha fijado ${ }^{6}$, en la respectiva audiencia, los puntos o hechos controvertidos que son el tema de prueba en el proceso judicial.

Las figuras 3, 4 y 5 ilustran la entrada de estos tres datos al sistema experto en un proceso verbal por nulidad absoluta de un contrato de compraventa por objeto ilícito y dolo7.

En dicho proceso, la pretensión principal ${ }^{8}$ que el demandante (vendedor) solicita al juez es que se declare la nulidad absoluta del contrato de compraventa por objeto ilícito y dolo por parte del demandado (comprador). Esta pretensión es planteada por el juez como una hipótesis que se afirma por el demandante (comprador) de la siguiente forma:

$4 \quad$ Artículo 196. Carga de la prueba.

Salvo disposición legal diferente, la carga de probar corresponde a quien afirma hechos que configuran su pretensión, o a quien los contradice alegando nuevos hechos.

5 Artículo 189 Código Procesal Civil del Perú. Oportunidad.

Los medios probatorios deben ser ofrecidos por las partes en los actos postulatorios, salvo disposición distinta de este Código.

6 El artículo 190 del Código Procesal Civil peruano, el cual prescribe la pertinencia e improcedencia de las pruebas, hace alusión a los hechos admitidos y controvertidos por las partes, y a las etapas probatorias de la proposición (demanda, contestación y reconvención) y de la ordenación (audiencia de fijación de puntos). Etapas en las que el juez extrae e ingresa (entradas) estos tres insumos al sistema experto Juez Inteligente.

7 Se propone el caso parcialmente, dado que el objetivo es ilustrar el funcionamiento del sistema experto.

8 Las demás pretensiones no son tenidas en el presente artículo, pues lo que se pretende es la ilustración del funcionamiento del sistema experto. 
Pretensión: La compraventa es nula por ilicitud y dolo.

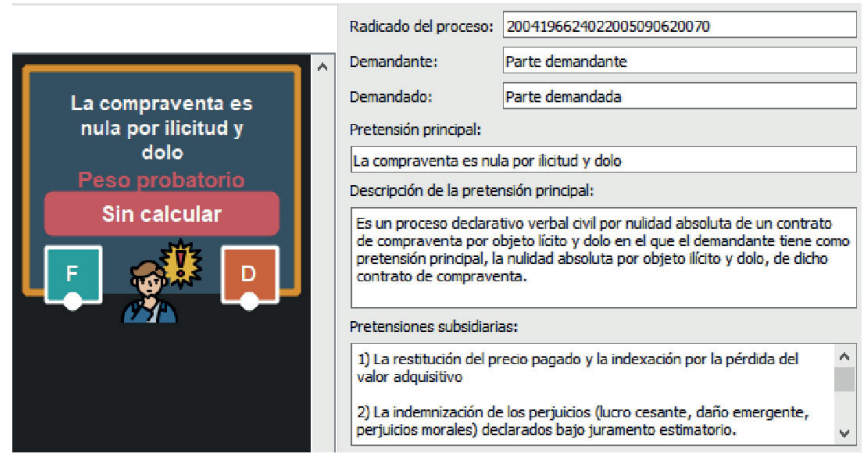

Figura 3(a). La pretensión principal del demandante (comprador).

Figura 3:

La pretensión principal del demandante (comprador). Fuente: Elaboración propia.

Los hechos que favorecen $(f)$ esta pretensión principal del demandante, son planteados como enunciados o proposiciones simples de la siguiente forma:

i. Primer hecho: El demandante compró por escritura.

Mediante escritura pública, el demandante compró al demandado el bien inmueble debidamente delimitado e identificado.

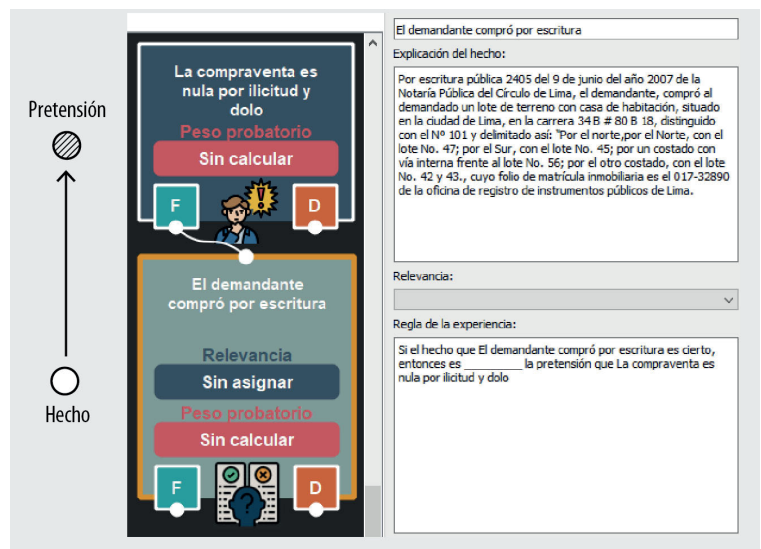

Figura 3(b). La pretensión principal y el hecho \# 1 del demandante (comprador).

Figura 4:

La pretensión principal y el hecho \# 1 del demandante (comprador). Fuente: Elaboración propia. 
ii. Segundo hecho: El demandante (comprador) no conocía la medida cautelar del bien.

El mencionado contrato de compraventa es nulo por objeto ilícito y dolo, dado que el inmueble vendido contaba con una medida cautelar de embargo decretada por un juzgado. Además, en tanto que el demandado (vendedor) actuó con una serie de argucias de mala fe que llevaron al comprador a realizar el contrato. Si el demandante (comprador) hubiere sabido de estas conductas delictivas del demandado (vendedor), entonces no hubiese realizado dicho contrato.

iii. Tercer hecho: No hubo autorización por parte del juez que decretó la medida cautelar del bien inmueble. Para concretar el contrato de compraventa jamás se autorizó por el juez que decretó las medidas cautelares de embargo, y mucho menos se contó con el consentimiento del acreedor.

Las pruebas que favorecen $(f)$ los hechos del demandante, y que son propuestas o aportadas en la demanda, son ingresadas de la siguiente forma:

i. Documentales

- Escritura pública \# 2405 del 9 de junio del año 2007.

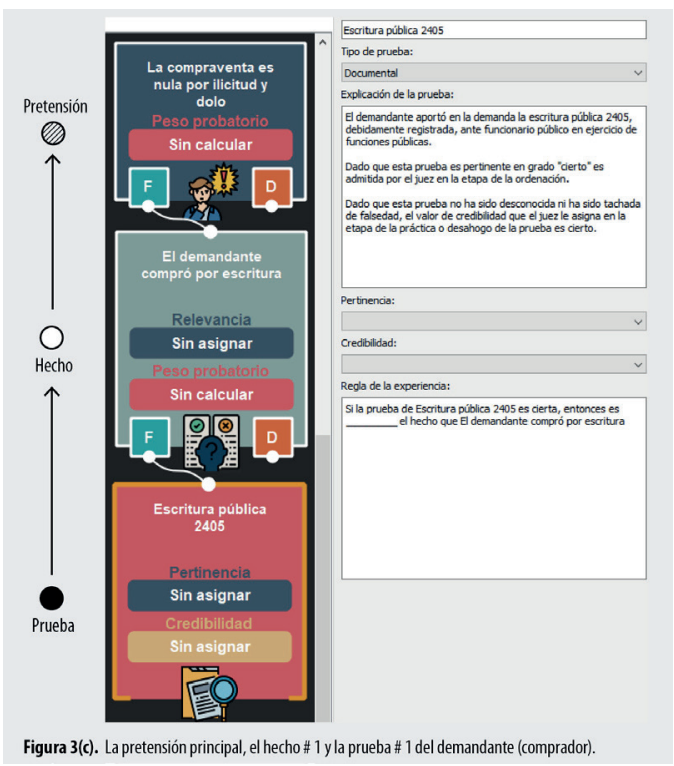

Figura 5:

La pretensión principal, el hecho \# 1 y la prueba \# 1 del demandante (comprador). Fuente: Elaboración propia. 
- Certificado de libertad y propiedad expedido por la oficina de registro de instrumentos públicos y privados.

- Oficio de la medida cautelar practicada.

- Constancia del juzgado, donde consta que nunca se solicitó la autorización de la venta del bien por parte del demandado (vendedor).

ii. Testimoniales

- Declaración de parte del demandado (vendedor).

- Testimonios de Juan Alzate y Mariana Ospina.

\section{CON RELACIÓN AL SEGUNDO HECHO, LOS TESTIGOS DARÁN CUENTA DE LOS ARGUMENTOS FALSOS DADOS POR EL DEMANDADO (VENDEDOR) EN RELACIÓN CON EL LEVANTAMIENTO DE LA MEDIDA CAUTELAR}

Credenciales probatorias y reglas de la experiencia y/o de la ciencia que deben ser asignadas por el usuario en el sistema experto Juez Inteligente

Las credenciales probatorias con las cuales opera el sistema experto Juez Inteligente son tres: i) relevancia-pertinencia, ii) credibilidad y iii) peso probatorio. Las reglas de la experiencia y de la ciencia son el "cemento" que permite el enlace o la conexión inferencial entre i) las pruebas y los hechos; ii) y los hechos y la pretensión (Schum, 2016).

\subsection{Relevancia - pertinencia}

Es el grado de conexión en términos de probabilidad que se presenta entre i) un hecho y una pretensión (relevancia), y ii) una prueba y un hecho (pertinencia). En la construcción de inferencias probatorias se parte de una pretensión hacia un hecho y luego de este hecho hacia una prueba. Relevancia y pertinencia son palabras sinónimas. En el derecho procesal y probatorio, la primera se usa para los hechos y la última para las pruebas.

\subsubsection{Relevancia de los hechos o hechos jurídicamente relevantes}

Es el grado de conexión en términos de probabilidad que se presenta entre un hecho y una pretensión. Es decir, si un hecho es cierto, entonces una pretensión será más o menos probable en un determinado grado o medida. En el caso actual, el primer hecho, hace más probable la pretensión del demandante (comprador) y, por tanto, es un hecho relevante. Esto es lo que hace que pueda 
hablarse de un hecho jurídicamente relevante o de la relevancia de un hecho en relación con una pretensión.

Para establecer la relevancia jurídica de un hecho el juez se pregunta: ¿cuán probable hace este hecho la pretensión? ¿cuán relevante es este hecho en relación con la pretensión? ¿nada probable? ¿poco probable? ¿probable? ¿muy probable?

Entonces, en la etapa de la actividad probatoria de la proposición, una vez que han sido fijados los puntos o hechos controvertidos, el juez define la relevancia jurídica de cada hecho en relación con la pretensión, asignando un valor de relevancia al mismo.

La figura 6 ilustra estas etapas de la actividad probatoria en el proceso judicial:

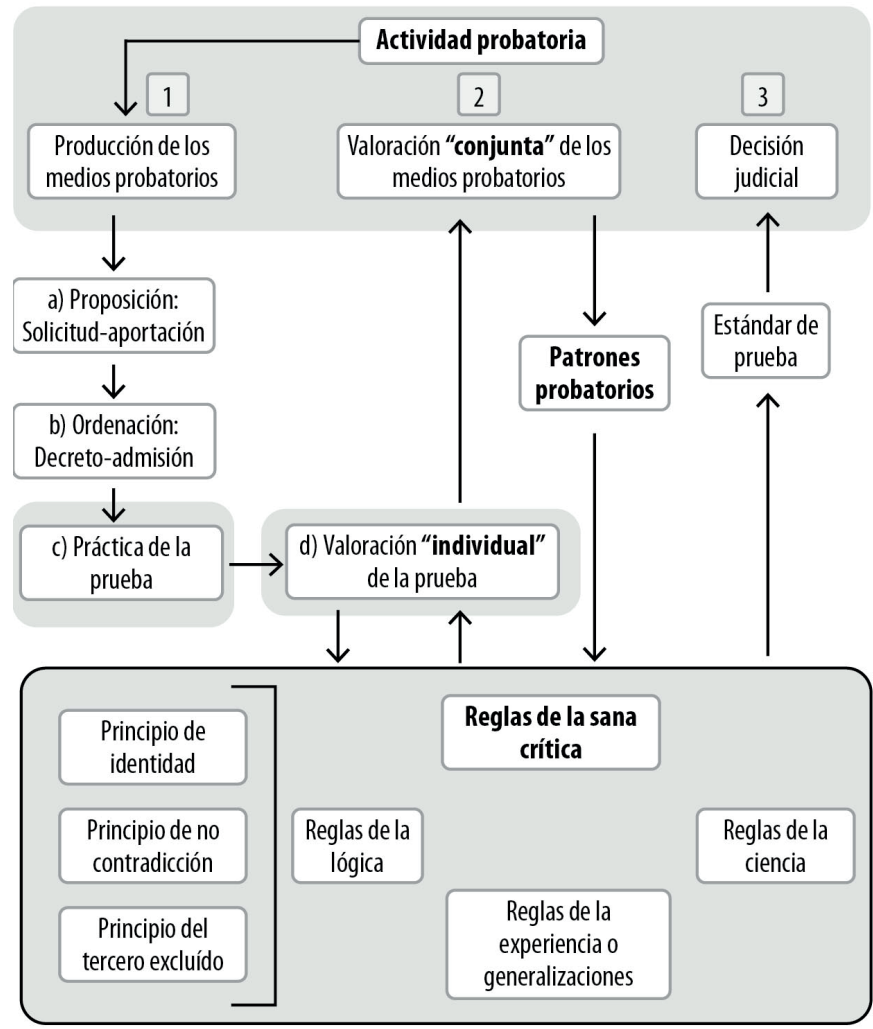

Figura 4. Las etapas de la actividad probatoria en el proceso judicial.

Figura 6:

Las etapas de la actividad probatoria en el proceso judicial. Fuente: Elaboración propia. 
En el presente caso, el primer hecho hace probable la pretensión. Es decir, el primer hecho es un hecho jurídicamente relevante en relación con la pretensión. El valor asignado por el juez a la relevancia de este primer hecho en relación con la pretensión es probable.

La figura 7 ilustra la asignación de este valor de pertinencia de probable al primer hecho en relación con la pretensión:

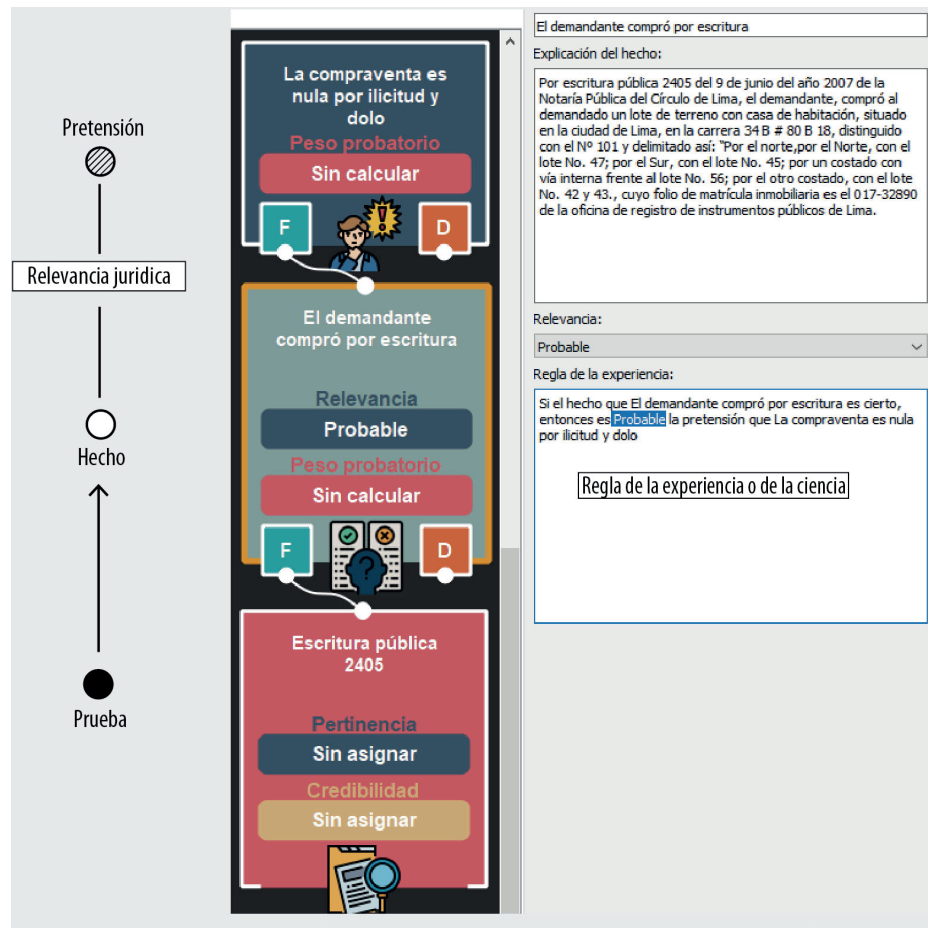

Figura 5. La relevancia del hecho en relación con la pretensión y su justificación mediante una regla de la experiencia.

Figura 7:

La relevancia del hecho en relación con la pretensió y su justificación mediante una regla de la experiencia. Fuente: Elaboración propia.

\subsubsection{Reglas de la experiencia y/o de la ciencia como justificación del valor de relevancia asignado a los hechos jurídicamente relevantes}

Para justificar el valor de relevancia asignado a los hechos jurídicamente relevantes, se hace uso de las reglas de la experiencia o reglas de la ciencia que hacen parte del sistema de valoración de la sana crítica consagrados en 
los artículos 197 del Código de Procedimiento Civil y 393 del Código de Procedimiento Penal del Perú (ver nota al pie 3 arriba).

Una regla de la experiencia o de la ciencia es una operación mental o inferencia inductiva que puede ser planteada como un enunciado cubierto probabilísticamente bajo la siguiente estructura: si un evento A es cierto (ocurre), entonces es probable que un evento B sea cierto (ocurra). Por ejemplo: $\mathrm{Si}$ es cierto que hay una fuga de gas en un lugar, entonces es probable que haya una explosión en dicho lugar.

En el caso actual, el sistema experto Juez Inteligente, construye la siguiente regla de la experiencia o de la ciencia, la cual, enlaza probabilísticamente el primer hecho con la pretensión (ver la figura 7 arriba): si es cierto que el demandante compró el bien por escritura pública, entonces es probable que dicha compraventa sea nula por ilicitud y dolo.

Esta regla de la experiencia justifica el valor de la relevancia jurídica del primer hecho en relación con la pretensión principal.

\subsubsection{Pertinencia de la prueba}

Es el grado de conexión en términos de probabilidad que se presenta entre una prueba y un hecho. En el caso actual, la prueba documental de la escritura pública 2405, hará más o menos probable el hecho que el demandante haya comprado por escritura.

Es decir, si la prueba de la escritura pública 2405 es cierta (auténtica), entonces el hecho que el demandante haya comprado por escritura será más o menos probable en un determinado grado o medida. Esto es lo que hace que pueda hablarse de la pertinencia de la prueba en relación con un hecho que es jurídicamente relevante.

Para establecer la pertinencia de la prueba el juez se pregunta: ¿cuán probable hace esta prueba este hecho? ¿cuán pertinente es esta prueba en relación con este hecho? ¿nada probable? ¿poco probable? ¿Probable? ¿muy probable? ¿cierto?

Entonces, en la etapa de la actividad probatoria de la ordenación (ver figura 6 arriba), el juez asigna un valor de pertinencia a cada prueba relacionada con un hecho jurídicamente relevante.

En el presente caso, la prueba documental de la escritura pública 2405 hace casi cierto el hecho que el demandado haya comprado por escritura. Es decir, la prueba de la escritura pública 2405 es pertinente en grado casi cierto en relación con el hecho que el demandante compró por escritura pública. El 
valor asignado por el juez a la pertinencia de esta prueba en relación con el hecho es casi cierto.

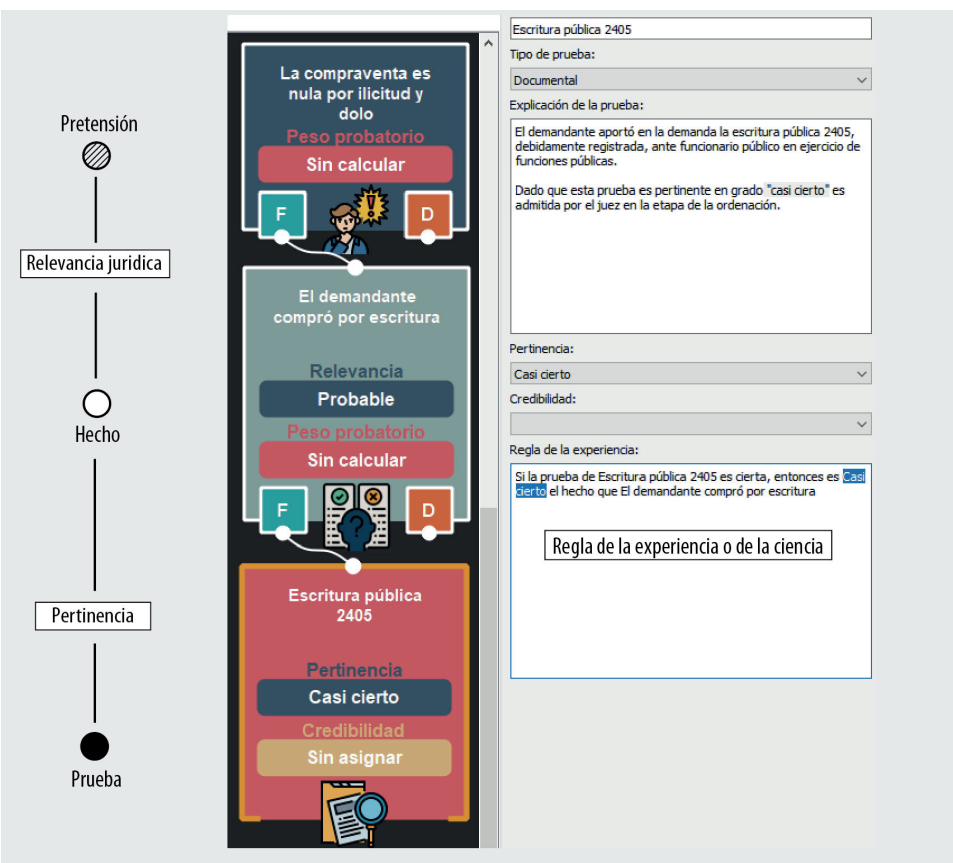

Figura 6. La pertinencia de la prueba en relación con el hecho. Justificación mediante una regla de la experiencia o de la ciencia.

Figura 8:

La pertinencia de la prueba en relación con el hecho. Justificación mediante una regla de la experiencia o de la ciencia. Fuente: Elaboración propia.

\subsubsection{Reglas de la experiencia o de la ciencia como justificación del valor de pertinencia asignado a las pruebas}

Análogamente, el valor de pertinencia asignado a las pruebas es justificado empleando reglas de la experiencia o de la ciencia.

En el caso actual, el sistema experto Juez Inteligente construye la siguiente regla de la experiencia, la cual enlaza probabilísticamente la prueba documental de la escritura pública 2405 con el hecho de que el demandante compró por escritura (ver la figura 8 arriba): si la prueba documental de la escritura pública 2405 es cierta (auténtica), entonces es casi cierto que el demandante compró por escritura pública. 
Esta regla de la experiencia justifica el valor de pertinencia asignado de casi cierto a la prueba documental de la escritura pública 2405 en relación con el hecho de que el demandante haya comprado por escritura.

Es importante señalar que hasta este momento se tiene un hecho jurídicamente relevante (relevancia = probable) con una prueba de tal hecho altamente pertinente $($ pertinencia $=$ casi cierto) .

\subsection{Credibilidad de la prueba}

La prueba documental de la escritura pública 2405 (como medio de prueba) se convierte en prueba (como resultado) una vez su pertinencia ha sido establecida en relación con el hecho que el demandante compró por escritura, lo cual debe ser probado por el demandante e intentar ser impugnado por el demandado.

En la escritura pública 2405, el juez puede leer que el demandante compró el bien inmueble. Que sea cierto o no el hecho de que el demandado compró por escritura pública es una inferencia y no un hecho. La distinción entre la prueba de un hecho y el hecho en sí, es fundamental en la construcción de las inferencias probatorias.

El hecho de que "el demandado compró por escritura" sea o no cierto, depende de la credibilidad de la prueba documental de la escritura pública 2405. De no considerar la autenticidad de esta prueba documental, se estaría suprimiendo cualquier duda razonable acerca de esta prueba documental ofrecida por el demandante.

Para establecer la credibilidad de la prueba el juez se pregunta: ¿cuán probable es que lo que dice esta prueba sea cierto? ¿cuán creíble es esta prueba en relación con este hecho? ¿nada probable? ¿poco probable? ¿probable? ¿muy probable? ¿casi cierto? ¿cierto?

Dado que desde la contestación de demanda la prueba documental de la escritura pública 2504 no fue desconocida ni tachada de falsedad por el demandado, el juez asigna un valor de credibilidad de cierto.

La figura 9 ilustra la asignación de este valor de credibilidad de cierto a la prueba en relación con el hecho de que "el demandante compró por escritura". También, la relevancia del hecho, la pertinencia y la credibilidad de la prueba. De igual forma, la regla de la experiencia que es empleada para justificar el valor de pertinencia asignado por el juez en la etapa de la ordenación a la prueba documental (escritura pública 2405). 


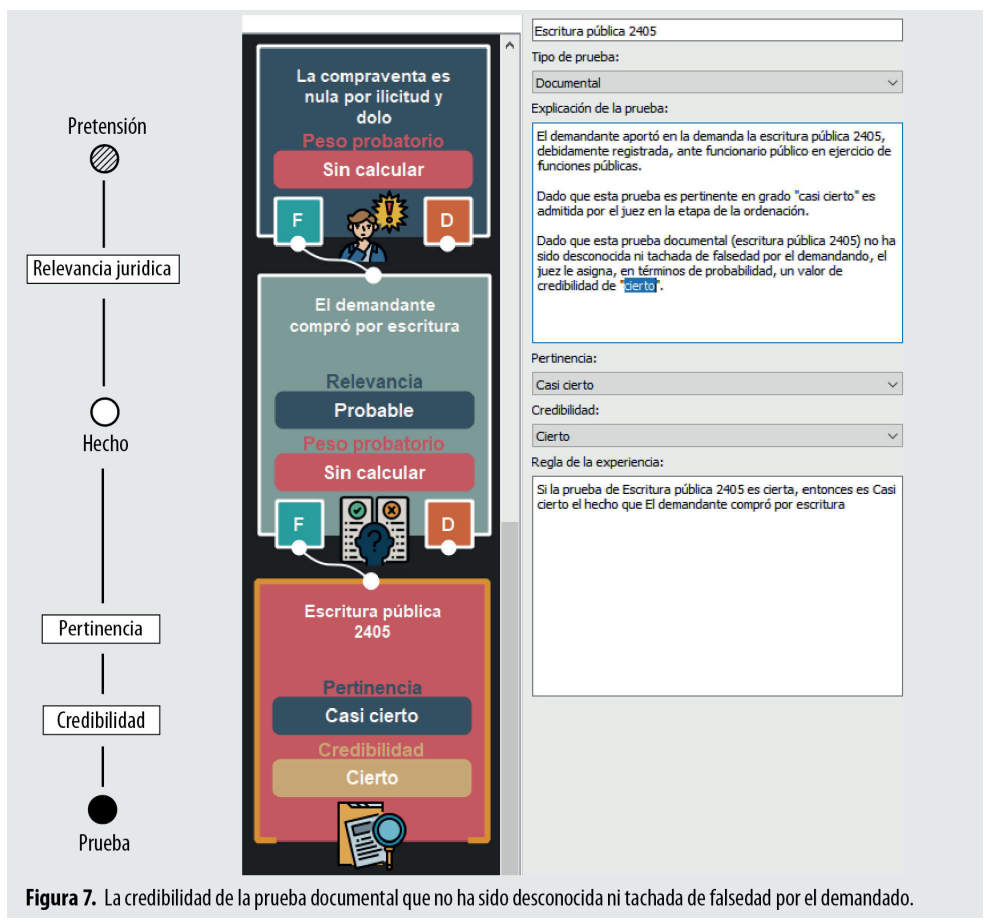

Figura 9

La credibilidad de la prueba documental que no ha sido desconocida ni tachada de falsedad por el demandado. Fuente: Elaboración propia.

\subsection{Peso probatorio de la prueba}

En el año 2017 realicé la cotraducción al castellano y publicación de la principal obra del filósofo británico L. J. Cohen, "Lo probable y lo demostrable", publicada originalmente en 1977 en la universidad de Oxford (Cohen, 2017).

En dicha obra, Cohen desarrolla un sistema de probabilidades relacionado con la inducción eliminatoria y variante. Este sistema de probabilidades, denominado baconiano, por su relación con las ideas de Francis Bacon (Bacon, 1949), se mueve entre dos extremos: a) falta de prueba y b) prueba.

\subsubsection{Falta de prueba}

Por un lado, este extremo significa que no se tiene prueba en relación con un determinado hecho que es jurídicamente relevante. Por esta razón, un hecho 
jurídicamente relevante, puede quedarse sin soporte o no estar soportado con base en pruebas disponibles.

Por ejemplo, si una persona confiesa ser titular del derecho real de dominio de un bien inmueble, dicha prueba testimonial es inconducente en relación con la prueba del hecho jurídicamente relevante de la titularidad del derecho real de dominio del bien inmueble. Por esta razón, un hecho que es jurídicamente relevante en un proceso civil, se puede quedar sin soporte inductivo dado que la prueba que pretende demostrarlo es inconducente.

En igual sentido, la prueba impertinente, inútil, ilegal o ilícita pueden igualmente dejar sin soporte un determinado hecho que es jurídicamente relevante en un determinado tipo de proceso. En general, para el sistema experto Juez Inteligente, si una prueba es inconducente, inútil, ilegal o ilícita, entonces es impertinente y, en consecuencia, deja sin soporte el hecho que con dicha prueba pretende ser probado.

Estos requisitos son la base con la cual el juez rechaza de plano dichas pruebas, es decir, no las admite ni decreta en la etapa de la ordenación, dejando sin soporte el hecho que pretende ser probado con las mismas.

\subsubsection{Prueba}

Por otro lado, este otro extremo significa que sí se tiene prueba en relación con un determinado hecho que es jurídicamente relevante. Por esta razón, un hecho jurídicamente relevante puede tener soporte inductivo en algún grado o medida con base en pruebas pertinentes, conducentes, útiles, legales y lícitas.

Las probabilidades baconianas propuestas por Cohen tienen propiedades ordinales (no cardinales). Esto significa que pueden ser comparadas, pero no combinadas algebraicamente acorde con la teoría de la probabilidad matemática.

En el sistema baconiano de probabilidades, la probabilidad inductiva de cero (0) asignada a un hecho significa falta de prueba ${ }^{9}$. En la medida en que sea encontrada alguna nueva prueba, este valor de probabilidad puede ser incrementado. La probabilidad de uno (1) significa que prueba del hecho y por ende el soporte probatorio estaría en su valor "máximo" (Cohen, 2017).

9 En el sistema pascaliano o matemático de probabilidades, si un hecho tiene un valor de probabilidad de cero (0), esto significa que el hecho no permite revisión alguna pues se trata de un hecho cuya ocurrencia es imposible. El valor de uno (1) significa que tenemos la certeza en relación con la ocurrencia del hecho. 
En el sistema baconiano de probabilidades, la pretensión de una de las partes puede ser más o menos probable que la pretensión de su contraparte. Cuando se afirma que la pretensión del demandante es más probable que la pretensión del demandado, esto significa que el demandante tiene una mayor variedad y número de pruebas pertinentes y creíbles que favorecen su pretensión de las que sustentan la pretensión del demandado (Keynes, 2013) (Cohen, 2017).

Lo que cuenta aquí es la variedad y número de pruebas pertinentes y creíbles (Cohen, 2017) que soportan los hechos jurídicamente relevantes sobre los cuales se fundamentan las pretensiones de las partes.

\section{ALGUNAS PROPIEDADES Y REGLAS DE LA PROBABILIDAD BACONIANA}

\subsection{Propiedad ordinal}

La pretensión del demandante con base en la prueba que trae al proceso puede ser mayor o igualmente probable que la pretensión del demandado con base la prueba que trae al proceso y viceversa.

Esto significa que, la probabilidad baconiana (PB) de la pretensión del demandante $\left(\mathrm{Pr}_{\mathrm{dte}}\right)$ con base en su prueba $\left(\mathrm{p}_{\mathrm{dte}}\right)$, puede ser mayor o igual que la probabilidad baconiana (PB) de la pretensión del demandado $\left(\mathrm{Pr}_{\text {ddo }}\right)$ con base en su prueba $\left(\mathrm{p}_{\mathrm{ddo}}\right)$, y viceversa. Dichas probabilidades pueden expresarse de la siguiente manera: $\mathrm{PB}\left(\mathrm{Pr}_{\mathrm{dte}} / \mathrm{p}_{\mathrm{dte}}\right) \geq \mathrm{PB}(\mathrm{Prddo} / \mathrm{pddo})$ O $\mathrm{PB}\left(\mathrm{Pr}_{\mathrm{ddo}} / \mathrm{p}_{\mathrm{ddo}}\right) \geq \mathrm{PB}$ $\left(\operatorname{Pr}_{\mathrm{dte}} / \mathrm{p}_{\mathrm{dte}}\right)$.

\subsection{Propiedad de la negación}

Si se tiene prueba del demandante (pdte) en relación con su pretensión $\mathrm{B}($ Prdte), y no se tiene prueba en contrario o que niegue la prueba del demandante (no-pdte) por parte del demandando, entonces la pretensión del demandado (Prddo), con base en la prueba del demandante (pdte), se queda sin soporte y es igual a 0 . Esto significa que la prueba del demandante (pdte) no puede soportar al mismo tiempo las pretensiones incompatibles del demandante (Prdte) y el demandado (Prddo) ${ }^{10}$.

10 Las probabilidades pascalianas o matemáticas (PM) para los eventos complementarios deben sumar uno (1) o el cien por ciento (100\%). PM (A) + PM (no-A) $=1=100 \%$. PM (Iluvia) + PM (no lluvia) $=1=100 \%$. Esta normalización que se corresponde en la teoría de la probabilidad con el principio complementario para la negación, no opera de la misma forma en el sistema baconiano de probabilidades propuesto por Cohen. 
Si PB (Prdte / pdte) $>0$ y PB (no-pdte) $=0$, entonces PB (Prddo $/$ pdte $)=0$

\subsection{Regla de la conjunción}

Si la probabilidad baconiana (PB) de la credibilidad (Credibilidad) de una prueba es mayor que la probabilidad baconiana (PB) de la pertinencia (Pertinencia) de dicha prueba, entonces la probabilidad baconiana (PB) de su conjunción (Credibilidad y Pertinencia) será la probabilidad con menor valor y viceversa (acorde con la propiedad ordinal mencionada arriba). Esto significa que, en el sistema baconiano propuesto por Cohen, la probabilidad baconiana de una conjunción nunca es menor que la probabilidad baconiana más pequeña de sus conjunciones.

Si PB (Credibilidad) > PB (Pertinencia), entonces PB (Credibilidad y Pertinencia $)=P B(\text { Pertinencia })^{11}$.

Si PB (Pertinencia) > PB (Credibilidad), entonces PB (Credibilidad y Pertinencia $)=P B($ Credibilidad $)$.

Esta regla permite calcular el peso probatorio que una prueba da a un hecho que es jurídicamente relevante. Para que una prueba pueda tener peso probatorio, sobre un hecho jurídicamente relevante, deberá ser al mismo tiempo creible y pertinente.

En la figura 9 arriba, si se tiene prueba documental totalmente auténtica (Cierto) y altamente pertinente (Casi cierto), entonces el peso probatorio que la misma da al hecho jurídicamente relevante que el vendedor compró por escritura, será el menor valor entre su credibilidad (Cierto) y su pertinencia (Casi cierto) $^{12}$. En este caso, el menor valor se corresponde con el de la perti-

11 En la figura 9 arriba se tiene un valor de credibilidad de Cierto y un valor de pertinencia de Casi cierto.

El peso probatorio que dicha prueba da al hecho sería el menor valor entre estos dos valores de credibilidad y pertinencia, es decir Casi cierto. La figura 10 ilustra el valor (Casi cierto) del peso probatorio que es calculado por el sistema experto Juez Inteligente. Si $P B(C)>P B(P)$, entonces PB (C y P) $=$ PB (P). Si PB (Cierto) > PB (Casi cierto), entonces PB (Cierto y Casi cierto) $=$ PB (Casi cierto).

12 En el sistema matemático o pascaliano de probabilidades (PM), acorde con el principio multiplicativo para la conjunción, la probabilidad matemática conjunta de dos hechos $\mathrm{A}$ y $\mathrm{B}$, viene dada por la siguiente expresión: PM (A y B) = PM (A) X PM (B/A); si los hechos A y B son independientes, entonces la expresión se convierte en: PM (A y B $=$ PM (A) X P(B). Por ejemplo, la probabilidad matemática (PM) conjunta de que llueva y haga sol, viene dada por la expresión: PM (Iluvia y sol) = PM (lluvia) X PM (sol). Si se asignan los valores de probabilidades para el hecho de la lluvia de PM (lluvia) $=0.9$ y para el hecho del sol de PM $($ sol $)=0.4$; entonces la probabilidad matemática 
nencia, es decir, el peso probatorio que esta prueba da al hecho es poco peso (Casi cierto).

De igual forma, esta regla permite calcular el peso probatorio que la prueba documental relacionada con el hecho jurídicamente relevante da a la pretensión. Dado que la prueba documental tiene un alto peso probatorio (Casi cierto) y el hecho es jurídicamente relevante (probable), el peso probatorio que esta prueba documental da a la pretensión, será el menor valor entre el peso probatorio (Casi cierto) que la prueba da al hecho y la relevancia del hecho (probable) en relación con la pretensión. Por tanto, el peso probatorio que esta prueba da a la pretensión es medio (Probable).

La figura 10, ilustra los dos pasos o eslabones inferenciales que emplea el sistema experto Juez Inteligente para el cálculo del peso probatorio que la prueba da a la pretensión. Un primer paso, desde la prueba hacia el hecho jurídicamente relevante y un segundo paso, desde el hecho jurídicamente relevante hacia la pretensión. En el primer paso, el juez asigna los valores de pertinencia y credibilidad a la prueba. En el segundo paso, el juez asigna el valor de relevancia al hecho. Con estos valores, el sistema experto Juez Inteligente, calcula el peso probatorio que la prueba individualmente considerada (valoración individual) da a la pretensión como Probable.

conjunta de que llueva y haga es expresada de la siguiente forma: PM (lluvia y sol) $=(0.9) X$ $(0.4)=(0.36)$.

De lo anterior se observa que, para la ocurrencia conjunta de los hechos independientes de la Iluvia y el sol, la multiplicación o conjunción de las probabilidades de dichos hechos independientes, siempre dará como resultado un valor menor que cualquiera de sus probabilidades consideradas por separado.

Es decir: $P(A$ y $B)<P(A)$, ya que $0.36<0.9$. Además, $P(A$ y $B)<P(B)$, dado que $0.36<0.4$.

La probabilidad baconiana $(\mathrm{PB})$ de una conjunción nunca es menor que la probabilidad baconiana más pequeña de sus conjunciones. El principio multiplicativo para la conjunción baconiana, puede ser visto como una regla de minimización (Min) entre los valores conjuntos de la credibilidad y la pertinencia de la prueba, y entre los valores del peso probatorio de la prueba y la relevancia del hecho relacionado con la pretensión. 


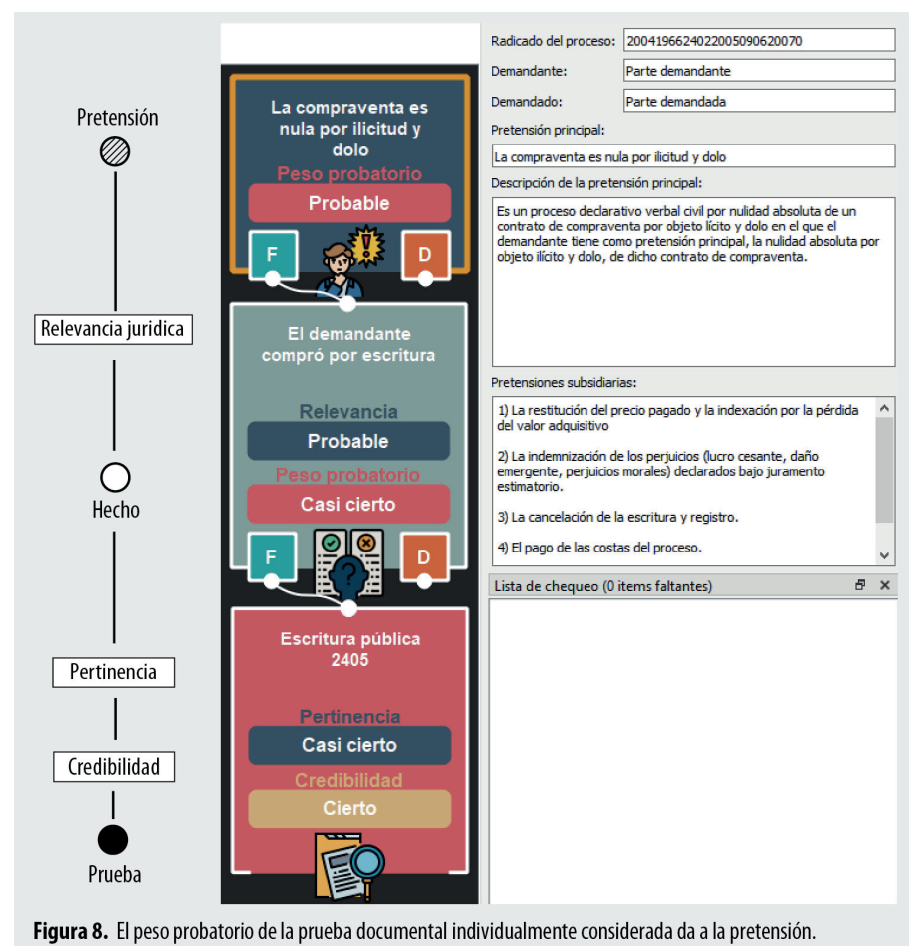

Figura 8. El peso probatorio de la prueba documental individualmente considerada da a la pretensión.

Figura 10:

El peso probatorio de la prueba documental individualmente considerada da a la pretensión. Fuente: Elaboración propia.

Los valores de probabilidad empleados en la figura 10, han sido propuestos por George Tecuci y varios de sus colegas en el centro de agentes de aprendizaje de la escuela de Ingeniería Volgenau de la universidad George Mason, en el desarrollo de diversos asistentes cognitivos para el análisis de inteligencia ${ }^{13}$ (Tecuci G., 2018). La escala de probabilidades propuesta por estos autores ha sido realizada combinando las probabilidades baconianas de L. J. Cohen y los cualificadores difusos propuestos por Lotfi Zadeh.

El sistema experto Juez Inteligente, emplea esta escala de probabilidades y los rangos numéricos asociados a dichas probabilidades, los cuales se ilustran a continuación

13 Disciple-LTA8-10, TIACRITIS11, Disciple-CD4 y una versión previa de Cogent. 
Sin soporte $(0-50 \%)<$ Poco probable $<(50-55 \%)<$ Probable $(55-70 \%)<$ Más probable $(70-80 \%)<$ Muy probable $(80-95 \%)<$ Casi cierto $(95-99 \%)<$ Cierto (100\%)

De acuerdo a esta tabla de probabilidades, en el sistema experto Juez Inteligente se establecen los diversos rangos para los estándares de prueba en materia civil y en materia penal (prueba preponderante, prueba clara y convincente, causa probable, la inferencia razonable, probabilidad de verdad y más allá de toda duda). La figura 11 ilustra esto ${ }^{14}$.

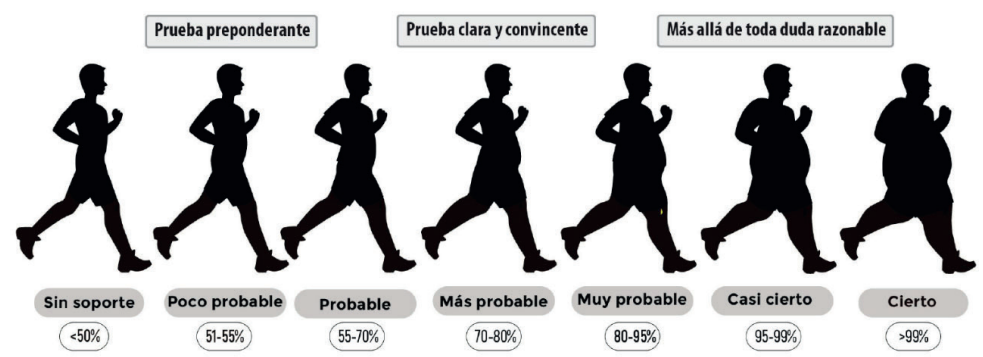

Figura 9. Escala de probabilidades y estándares de prueba.

Figura 11:

Escala de probabilidades y estándares de prueba.Fuente: Elaboración propia.

\subsection{Regla de la disyunción}

Cuando dos o más pruebas favorecen o apuntan en la misma dirección de un hecho jurídicamente relevante, se presenta un patrón probatorio de corroboración probatoria. El peso probatorio conjunto que estas pruebas dan al hecho jurídicamente relevante, vendrá dado por el mayor valor de sus disyunciones. De igual forma, cuando dos o más pruebas favorecen o apuntan en dirección de hechos jurídicamente relevantes que siendo diferentes, apuntan en la dirección de la misma pretensión, se presenta un patrón probatorio de convergencia probatoria. El peso probatorio conjunto que estas pruebas dan a la pretensión, vendrá dado por el mayor valor de sus disyunciones. Esto significa que, en el sistema baconiano propuesto por Cohen, la probabilidad baconiana de una disyunción nunca es menor que la probabilidad baconiana más grande de sus disyunciones. 
La figura 12, ilustra estos dos patrones probatorios de corroboración y convergencia.

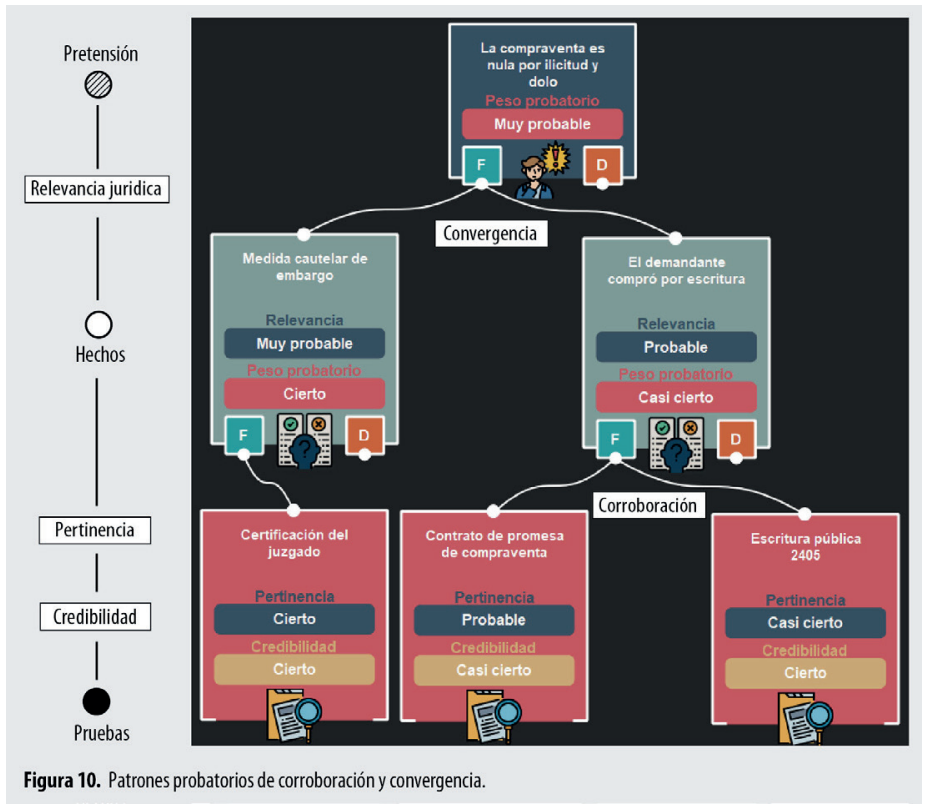

Figura 12:

Patrones probatorios de corroboración y convergencia. Fuente: Elaboración propia.

Si hay prueba que favorece un hecho y prueba que lo desfavorece, se presenta un patrón probatorio de contradicción probatoria. El peso probatorio conjunto que estas pruebas contradictorias dan al hecho jurídicamente relevante viene dado por una función de equilibrio ${ }^{15}$ creada al igual que la escala de probabilidades por George Tecuci y sus colegas (Tecuci, Schum et al., 2016).

De igual forma, si hay pruebas que favorecen hechos diferentes, de las cuales, unas favorecen la pretensión del demandante y otras favorecen la

15 On balance funtion. En esta función de equilibrio si la prueba favorable al hecho o pretensión tiene una probabilidad mayor que la prueba desfavorable al hecho o pretensión, entonces el nivel de la prueba favorable es disminuido en un grado en la escala de probabilidades. Si la prueba favorable al hecho o pretensión tiene una probabilidad menor o igual que la prueba desfavorable al hecho o pretensión, entonces el hecho o pretensión del demandante se queda sin soporte en la escala de probabilidades. 
pretensión del demandado, entonces se presenta un patrón probatorio de conflicto probatorio. La función de equilibrio da cuenta igualmente del peso probatorio de estas pruebas conflictivas.

La figura 13, ilustra estos cuatro patrones probatorios de corroboración, convergencia contradicción y conflicto.

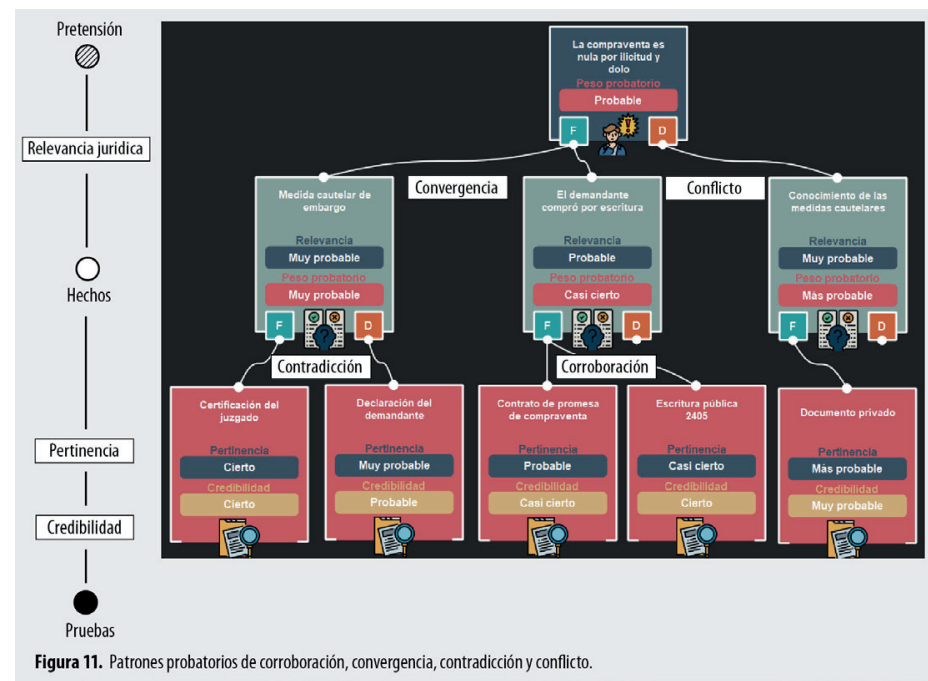

Figura 13:

Patrones probatorios de corroboración, convergencia, contradicción y conflicto. Fuente: Elaboración propia.

\subsection{Juez Inteligente}

Este escrito presenta una aproximación del sistema experto Juez Inteligente, el cual, asiste al estudiante y profesor de derecho, al abogado en ejercicio, al juez y al fiscal, en la construcción ordenada de inferencias probatorias. La valoración de la prueba judicial, que no es otra cosa que el razonamiento probatorio empleado en el proceso de toma de decisiones, puede ser comprendido y realizado de tal forma que los esfuerzos que son realizados por las personas que emplean pruebas, puedan ser dirigidos racional y razonadamente en los contextos de incertidumbre, donde la prueba se presenta de forma incompleta, ambigua, contradictoria e inexistente. Es mi deseo que este sistema experto pueda llegar a tener vida en Perú y por supuesto en otras latitudes latinoamericanas. 


\section{LISTA DE REFERENCIAS}

Anderson, T., Schum, D. A., \& Twining, W. L. (2005). Analysis of Evidence. Cambridge University Press. https://doi.org/10.1017/СBO9780511610585

Bacon, F. (1949). Novum Organon (C. H. Balmori, trad., e introducción y notas de R. Frondizi). Losada.

Cohen, L. J. (2017). Lo probable y lo demostrable (1.a ed., vol. 1) (O. Vargas y C. Ruiz, trad. y O. Vargas, ed.). Orión Vargas.

Houdé, O. (2003). Diccionario de ciencias cognitivas: neurociencia, psicología, inteligencias artificial, lingüística y filosofía. Amorrortu.

Keynes, J. M. (2013). A Treatise on Probability. Dover Publications.

Schum, D. A. (2016). Los fundamentos probatorios del razonamiento probabilístico (2.. ed.) (O. Vargas, ed. y trad.). Orión Vargas.

Tecuci, G., Marcu, D., Boicu, M., \& Schum, D. A. (2016a). Knowledge Engineering: Building Cognitive Assistants for Evidence-based Reasoning. Cambridge University Press. https://doi.org/10.1017/CBO9781316388464

Tecuci, G., Schum, D. A., Marcu, D., \& Boicu, M. (2016b). Intelligence Analysis as Discovery of Evidence, Hypotheses, and Arguments: Connecting the Dots. Cambridge University Press. https://doi.org/10.1017/CBO9781316388488

Tecuci, G., Kaiser, L., Marcu, D., Uttamsingh, C. \& Boicu, M. (2018). Evidence-based Reasoning in Intelligence Analysis: Structured Methodology and System. Computing in Science and Engineering, 20(6), 9-21.

https://doi.org/10.1109/MCSE.2018.2873852

Vargas, O. (2019). El razonamiento inductivo en la valoración de la prueba judicial. Ediciones Universidad de Salamanca.

Wigmore, J. H. (1937). The science of judicial proof, as given by logic, psychology, and general experience, and illustrated in judicial trials. Little, Brown. 